\title{
Modeling Strategic Location Choices for Disadvantaged Firms
}

\author{
Hejun Zhuang ${ }^{1}$ \\ ${ }^{1}$ Assistant Professor of Marketing, Business Administration Department, School of Art, Brandon University, \\ Manitoba, Canada \\ Correspondence: Hejun Zhuang, Business Administration Department, School of Art, Brandon University, \\ Brandon, Manitoba, R7A 6A9, Canada.
}

Received: August 22, 2018

doi:10.5539/ibr.v11n10p59
Accepted: September 8, 2018

Online Published: September 20, 2018

URL: https://doi.org/10.5539/ibr.v11n10p59

\begin{abstract}
This paper models how a firm's capability relative to that of the other firm affects his location choice in the marketplace. Weaker firms strategically avoid head-to-head competition with stronger ones. When the capability gap is small, weaker firms randomly visit the core market of competitors (the "dodge" strategy). By doing so, they can trigger competitors to leave the demands of boundary markets in order to defend their core markets. When the capability gap is medium, they focus their resources on niches to fight for survival (the "niche" strategy). These strategies differ from those of stronger firms, which defend on core markets when the capability gap is small and build new markets when the capability gap becomes larger. Results show that those location choices can be understood using game theoretical models - the Hotelling model and the Colonel Blotto game. The paper's results also explain the empirical observation that small businesses are more likely than large firms to make radical investments in $\mathrm{R} \& \mathrm{D}$.
\end{abstract}

Keywords: Colonel Blotto game, Hotelling model, location model, marketing strategy, SME

\section{Introduction}

Owing to their well-known brands, large advertising budgets, and strong management teams, big businesses like Amazon, IBM and Coca-Cola attract more consumers than other businesses. When competing with such giants in the market, how should weaker firms make their marketing strategies, in particular location choices, either geographically (e.g., the locations of physical stores) or conceptually (e.g., target audiences for ads or design of product features)? A common view, often serving as the basis for case studies, is that success can be copied, so firms can grow by following the similar operations and strategies of successful firms. The opposite view is that weaker firms should evade competition by seeking market niches. Can copying or differentiating from stronger firms' strategies help weaker firms break through market clutter? The literature still lacks theoretical verification. This paper studies this particular aspect of marketing strategy - firms' location choices when their capabilities in the marketplace are different. Game-theoretical models can also explain the observation that small businesses are more likely to make radical investments in R\&D than large firms.

This paper models how firms' relative capability difference impacts the location choices in the market place. Weaker firms strategically avoid head-to-head competition with stronger ones. When the capability gap is small, weaker firms randomly visit the core market of competitors (the "dodge" strategy). By doing so, they can trigger competitors to leave the demands of boundary markets to defend their core markets. When the capability gap is medium, they focus their resources on niches to fight for survival (the "niche" strategy). These strategies differ from those of stronger firms, which defend core markets when the capability gap is small and build new markets when the capability gap becomes larger. These location choices can be understood using game theoretical models the Hotelling model and the Colonel Blotto game.

As the capability gap is likely not the only factor influencing firms' adoption of location strategies, additional aspects of model-setup and assumptions that are not captured in this study's models, such as alternative setting on consumer distribution and firms' capability, are discussed in future research. We view our paper as an interesting start which helps us understand the empirical patterns observed in reality.

For managers, this paper provides guidelines for SMEs to enhance their market - an important contribution given the prevalence of SMEs. In 2010, small businesses made up $99.7 \%$ of U.S. employer firms, generating $64 \%$ of new private-sector jobs and $49.2 \%$ of private-sector employment. Furthermore, small firms spend almost twice as much 
of their R\&D budget on radical research as do large firms, and are roughly thirteen times more innovative per employee than large firms (Kobe, 2012). The National Science Foundation reported that small firms provide 24 times more innovation per research dollar than do large firms (Tornatzsky, 1983).

In examining the strategic interaction between parties with asymmetric capability, this study also sheds light on areas such as political elections, career choices, university positions, and sports. For example, in the study of the public candidate elections, scholars have found that weaker candidates move away from the political center to the left or right while the stronger candidates do the opposite, and also that challengers tend to adopt more extreme positions than incumbents (Ansolabehere, Snyder, \& Stewart, 2001).

\section{Related Research}

Location choice refers to firms (re)position themselves in the market on the basis of market features and organizational resources, involving targeting and differentiating to decrease direct competition and avoid competing on price only (Porter, 1980). An extensive literature in marketing and economics has examined positioning both empirically and theoretically.

Symmetric Firms. In empirical studies, researchers have studied diverse sets of issues: the determining factors in firms' location choices (Berry, 1992, 1994; Mazzeo, 2002; Seim, 2006; Watson, 2009), geographic distances between McDonald's and Burger King outlets (Thomadsen, 2007), the distribution of gasoline stations in the Los Angeles area (Netz \& Taylor, 2002), and the design of new car models in US automobile industry (Thomas \& Weigelt, 2000). Theoretical papers can be traced back to the pioneering work of Hotelling (1929), where customers are uniformly distributed in the market and two symmetric firms need to decide their market locations. He showed the Principle of Minimum Differentiation, where two competing firms choose to stay at the center of the market. Tirole (1988) provided a detailed summary of one-dimensional location models in which products are differentiated either horizontally (e.g., product quality) or vertically (e.g., product features). Researchers have also explored two-dimensional location models with both horizontal and vertical differentiation (Economides, 1986; De Palma, Ginsburgh, Papageorgiou, \& Thisse, 1985; Vandenbosh \& Weinberg, 1995), finding that firms tend to adopt a MaxMin product differentiation - maximize one dimension but minimize the other.

Asymmetric Firms. Most empirical location studies on asymmetric firms deal with entry models. For example, Carpenter and Nakamoto (1990) studied the optimal positioning, advertising, and pricing strategies for an entrant when the market is already dominated by a stronger incumbent. They found that owing to consumers' asymmetric preferences, "me-too" strategies should not be adopted as entry strategies. Theoretical location studies on entry models are also limited. If the incumbent is stronger than the entrant, the incumbent could pre-empt the most desirable product position and gain a first-mover advantage (Moorthy, 1988). However, if an incumbent is the weaker one (e.g., the second-mover enters with lower production costs), the incumbent should leave the most attractive location in the market and move to a market niche, and the larger the second-mover's cost advantage, the farther the first-mover should locate (Tyagi, 2000). On the other hand, in studying an entrant's location model, Seyman, Hoch, and Raju (2002) found that when a retailer wants to introduce its store brand and already sells two national brands, the appropriate perceptual market location of the store brand is close to that of the leading national brand. However, Du, Lee, and Staelin (2005) found the opposite result - that store brand should be positioned close to the weaker national brand.

Other than studies proposing entry models, studies on asymmetric firms' location decision are rare. Budd, Harris and Vickers (1993) adopted a stochastic model to model the dynamic change of firms' capability gap, which could be adjusted via firms' input of efforts along the way. They found that firms' capability gap expanded asymptotically, and under certain conditions the weaker firm input more effort.

Marketing and Military Strategy. The Colonel Blotto game is one of military strategies, in which two colonels are tasked to simultaneously send their troops over several battlefields, and the player with more troops in a battlefield wins that field. The payoff is equal to the number of fields won (Appendix A gives a detailed description of the game). The Colonel Blotto game has been applied to solve resource allocation problems not only in wars, electoral competitions, and tournaments (Groserclose, 2001; Krasa \& Polborn, 2010), but also in business, such as auctions. For example, Bayes, Kovenock and Viries (1996) constructed a Colonel Blotto model for a (first price) all-pay auction, in which bidders simultaneously submitted bids for an item and all players forfeited their bids. Likewise, Szentes and Rosenthal (2003) adopted the model to find symmetric equilibria for a specific sealed-bid auction, where two bidders bid for three identical objects and the objects' marginal valuations decrease.

The Colonel Blotto strategy was introduced to business and marketing literature by Friedman (1958), Trout and Ries (1978) and Kotler and Singh (1981), where market share is analogous to the number of battlefields and 
market competition to military conflicts. The technical difficulty in solving this problem has limited its wider applications. For some time, economists have been dedicated to seeking efficient solutions of this game. The first solution was provided by Borel and Ville (1938). Later, by using the properties of regular $n$-gons, Gross and Wagner (1950) generalized Borel's two solutions to the case of two players with symmetric forces. Recently, Roberson (2006) provided a feasible method for constructing a mixed equilibrium of n-variate distributions.

In summary, this study enriches location literature in several ways. First, it applies game-theoretical models to the strategic location decisions for asymmetric firms, an extension to the seminal works of classic IO models such as Hotelling (1929) and Salop (1979), which examined symmetric firms' location decisions. Second, the study addresses a gap in the location strategies literature, as despite numerous empirical studies, most theoretical modeling focuses on the competition among symmetric firms or entry models. A rigorous theoretical study on asymmetric firms' location strategies is still missing. Third, this study introduces the Colonel Blotto game, the competitive resource deployment in the battlefield (military strategy), to solve firms' location choices in the market place (marketing strategy).

\section{Model}

This section presents the model setup and the rationale for assumptions. Consider two asymmetric firms (Firm $\mathrm{H}$ and Firm L) indexed by $j$ (where $j=H, L$ ), carrying out a homogenous project. Firms need to choose optimal market locations for their projects, either geographically (e.g., the location of store) or conceptually (e.g., the product features of new product, or the target audiences of ads). The two firms differ in terms of factors such as strong reputation and/or better budget. Firm $\mathrm{H}$ attracts more consumer attention than Firm $\mathrm{L}$ - a capability gap between the firms denoted by $\eta$. At one extreme where $\eta$ is zero, the two firms are symmetric, and thus the location choice model reduces to the standard Hotelling model, in which both firms choose to locate at the market's center. At another extreme where $\eta$ is sufficiently large, Firm $\mathrm{H}$ captures the whole market, driving Firm L out of market. Therefore, this study excludes both extremes and focuses on the cases where $\eta$ is small or medium, so that strategic interactions exist between firms with respect to location choices, for example, brand positioning of Pepsi and Coca-Cola, airline route choices of major airlines, and geographic choices of McDonald's and Burger King outlets in the same city.

The market is comprised of $m$ consumers, uniformly distributed in a linear market of $[0,1]$ with one consumer in one of $m$ mutually exclusive and independent locations of $\{0,1 /(m-1), 2 /(m-1), \ldots,(m-2) /(m-1), 1\}$. The locations of consumers reflect their differences in tastes. This positioning is also known as horizontal differentiation. Consumers vote for firms through their attention, such as visiting the store, buying the firm's stock, or viewing the firm's advertising. Consumer $i^{\prime} s$ preference $u_{i}$ for Firm $\mathrm{H}$ can be expressed as $u_{i}=\eta_{0}+$ $\eta-\left(x_{i}-x_{H}\right)^{2}$, and for Firm L $u_{i}=\eta_{0}-\left(x_{i}-x_{L}\right)^{2}$, where $x_{H}, x_{L}$ and $x_{i}$ are Firm H's, Firm L's and Consumer $i$ 's locations, respectively; $\eta_{0}$ is the basic interest and $\eta$ is an additional attraction to Firm $\mathrm{H}$. We assume that $\eta_{0}>0$ and $\eta>0$. The disutility of a mismatch between consumer $i$ 's taste and the project of Firm $j$ is measured by the Euclidean distance between Consumer $i$ 's and Firm $j$ 's locations, $\left(x_{i}-x_{j}\right)^{2}$.

A game-theoretical approach can be used to solve firms' location $\left(x_{H}, x_{L}\right)$ choice in the linear market of $[0,1]$. This study looks for the firms' symmetric location strategies, as the sums of consumers' attention to firms remain unchanged if both firms' locations flip along the market center.

Proposition 1. When the capability between firms is small or medium, no pure equilibrium exists (see Appendix B for complete proofs).

The rationale is as follows. Suppose the pure strategy exists. Then both firms' optimal location choices are fixed points and can be predicted. As a result, Firm $\mathrm{H}$ will deviate and move to Firm L's location. Under the same distances from both firms, all consumers will vote for Firm H. Knowing this, Firm L will deviate and randomize its location to avoid being predicted by Firm H. The optimal location is thus not stable and the pure strategy does not exist. Next of interest is the mixed strategy, denoting Firm $j^{\prime}$ s location strategy by $\sigma^{j}=\left(\sigma_{1}^{j}, \ldots, \sigma_{m}^{j}\right)$.

Firm $j^{\prime}$ s attraction, denoted by $T_{j}(a, b)$ when Firm $\mathrm{H}$ and Firm L locate at $a$ and $b$, respectively, can be expressed as follows (Note 1):

$$
\begin{gathered}
T_{H}(a, b)=\left\{\begin{array}{cc}
m-a+1+\left[\frac{a-b-1}{2}\right] & a>b \\
m & a=b \\
a+\left[\frac{b-a-1}{2}\right] & b>a
\end{array}\right. \\
T_{L}(a, b)=m-T_{H}(a, b) .
\end{gathered}
$$


Equations (1) and (2) show a natural symmetry in the location strategy: firms' attractions remain unchanged after they flip their locations along the market center. Consequently, symmetry is expected in the mixed strategy. That is, $\sigma_{1}^{j}=\sigma_{m}^{j}, \sigma_{2}^{j}=\sigma_{m-1}^{j}, \ldots, \sigma_{\left[\frac{m}{2}\right]}^{j}=\sigma_{\left[\frac{m}{2}\right]+1}^{j}$ for $m>4$ and $m$ is even; $\sigma_{1}^{j}=\sigma_{m}^{j}, \sigma_{2}^{j}=\sigma_{m-1}^{j}, \ldots, \sigma_{\left[\frac{m}{2}\right]}^{j}=\sigma_{m-\left[\frac{m}{2}\right]+1}^{j}$ for $m>4$ and $m$ is odd.

Furthermore, firms' choice sets may not cover the entire market. Let $x_{k}^{j}$ be the starting location in Firm $j$ 's strategy, and the symmetric property of the strategy indicates that the last location in Firm $j$ 's strategy is $x_{m-k+1}^{j}$. An important feature of any mixed strategy equilibrium is that, given the market strategies chosen by the other players, each player is indifferent in choosing among any actions selected with positive probability. That is, within their market coverage/support set of $\left\{x_{k}^{j}, \ldots, x_{m-k+1}^{j}\right\}$, Firm $j$ 's attractions to consumers are the same.

\subsection{An Illustration}

First consider an analytical example for the features of the results. Let market size $m$ (i.e., the number of consumers) be six and these consumers are located at one of locations $\{1,2,3,4,5,6\}$ in the market (see Figure 1). (Note 2)

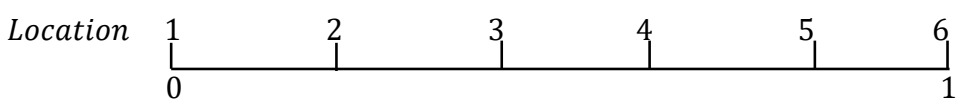

Figure 1. Consumers' location in the market when market size is six

\subsubsection{Small Capability Gap}

Consider the case where the capability gap is small, specifically, $0<\eta<\frac{1}{m-1}$. That is, consumers choose a better-matched firm, and prefer Firm $\mathrm{H}$ when matches from both firms are the same. Based on Equation (1) and (2), Firm H's attraction when locating at 3, given Firm L's location strategy of $\sigma^{L}=\left(\sigma_{1}^{L}, \ldots, \sigma_{6}^{L}\right)$, can be written as:

$$
T_{H}\left(3, \sigma^{L}\right)=5 \sigma_{1}^{L}+4 \sigma_{2}^{L}+6 \sigma_{3}^{L}+3 \sigma_{4}^{L}+4 \sigma_{5}^{L}+4 \sigma_{6}^{L} .
$$

In Equation (3), the first term is Firm H's attraction if Firm L locates at 1: Firm $\mathrm{H}$ wins over five consumers (i.e., three consumers who locate on its right, one consumer who locates on Firm H's location, and one consumer who locates between the two firms); five is multiplied by $\sigma_{1}^{L}$, because the probability of Firm L visiting Location 1 is $\sigma_{1}^{L}$. The second term is Firm H's attraction if Firm L locates at 2: Firm H wins over four consumers (i.e., three consumers who locate on its right, and one consumer who locates on its location); four is multiplied by $\sigma_{2}^{L}$, the probability of Firm L visiting Location 2. Similar arguments are applied to the third, fourth, fifth and sixth terms. Similarly, Firm H's attractions if choosing other locations, given Firm L's strategy of $\sigma^{L}$, can be expressed as follows:

$$
\begin{gathered}
T_{H}\left(1, \sigma^{L}\right)=6 \sigma_{1}^{L}+\sigma_{2}^{L}+2 \sigma_{3}^{L}+2 \sigma_{4}^{L}+3 \sigma_{5}^{L}+3 \sigma_{6}^{L}, \\
T_{H}\left(2, \sigma^{L}\right)=5 \sigma_{1}^{L}+6 \sigma_{2}^{L}+2 \sigma_{3}^{L}+3 \sigma_{4}^{L}+3 \sigma_{5}^{L}+4 \sigma_{6}^{L} .
\end{gathered}
$$

The equations when Firm H stays in the other side of market's center are saved owing to the symmetricity. The next is to narrow down firms' market coverage. Let Firm $j$ 's market coverage denoted by $\left\{x_{k}^{j}, \ldots, x_{m-k+1}^{j}\right\}$. Then the firm's attractions in its market coverage shall be equal. If Location 1 is the starting point of Firm H's strategy set, then Equations (3), (4) and (5) are equal, and the strategies are symmetric (i.e., $\sigma_{1}^{j}=\sigma_{6}^{j}, \sigma_{2}^{j}=\sigma_{5}^{j}, \sigma_{3}^{j}=\sigma_{4}^{j}$ ) for $j$ $=\{H, L\}$. Those lead to $\sigma_{1}^{L}=\cdots=\sigma_{6}^{L}=0$. Therefore, Location 1 shall be excluded from Firm H's strategy set. Going through similar arguments, Location 1 shall be also excluded from Firm L's market coverage.

Suppose that Location 2 is the starting point of Firm H's market coverage. Then $T_{H}\left(2, \sigma^{L}\right)=\cdots=\pi_{H}\left(5, \sigma^{L}\right)$, leading to $-\sigma_{2}^{L}+4 \sigma_{3}^{L}=0$. Because $\sum_{i=1}^{6} \sigma_{i}^{L}=1$ and because of the symmetricity of the strategy, $\sigma^{L}=$ 
$(0,0.4,0.1,0.1,0.4,0)$. Similarly, $\sigma^{H}=(0,0.1,0.4,0.4,0.1,0)$, firms' attractions $T_{H}=4.1$, and $T_{L}=1.9$.

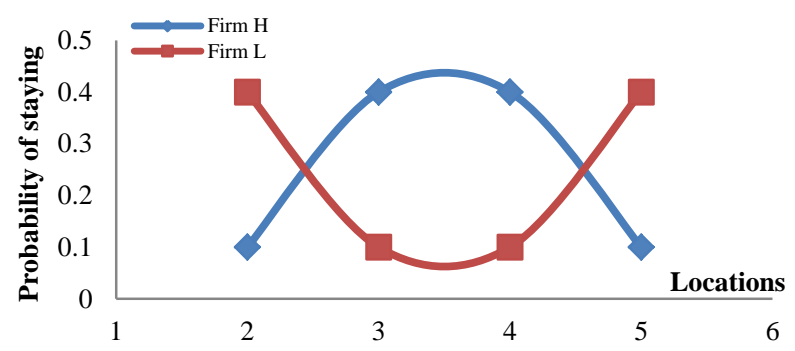

Figure 2. Firms' location strategies when the capability gap is small

Figure 2 depicts the mixed strategies. When the capability gap is small, the two firms choose the same locations but adopt different probabilities of staying on those locations. Firm $\mathrm{H}$ stays four times more on the center than on two edges. However, Firm L stays four times more on the edges than on the market center. The optimal strategy of Firm $\mathrm{L}$ is randomly visiting the market center to cause Firm $\mathrm{H}$ to invest more on the center to defend its core markets. In this way, Firm H leaves the demand of the boundary market to Firm L. The strategies of Firm H and Firm L are termed "defending" and "dodging," respectively.

\subsubsection{Medium Capability Gap}

Next, consider the case where the capacity gap is medium, specifically, $\eta \in\left[\frac{1}{m-1}, \frac{2}{m-1}\right)$, under which Firm $\mathrm{H}$ still wins the consumer if it stays only one more unit of location away from that consumer than does Firm L.

Note $\frac{1}{m-1}$ is one unit of location- that is, the distance of two closest locations. Here, six consumers locate as in

Figure 1, so the unit of location is $1 / 5$. The two firms need to decide their location strategies.

Based on Equations (1) and (2), a firm's attractions $T_{j}\left(x, \sigma^{L}\right)$ when staying at $x$, given the other firm's location strategy $\sigma^{j}$, can be written as follows (in the same manner of deriving Equation 3):

$$
\begin{gathered}
T_{H}\left(1, \sigma^{L}\right)=6 \sigma_{1}^{L}+2 \sigma_{2}^{L}+2 \sigma_{3}^{L}+3 \sigma_{4}^{L}+3 \sigma_{5}^{L}+4 \sigma_{6}^{L}, \\
T_{H}\left(2, \sigma^{L}\right)=6 \sigma_{1}^{L}+6 \sigma_{2}^{L}+6 \sigma_{3}^{L}+3 \sigma_{4}^{L}+4 \sigma_{5}^{L}+4 \sigma_{6}^{L}, \\
T_{H}\left(3, \sigma^{L}\right)=5 \sigma_{1}^{L}+6 \sigma_{2}^{L}+6 \sigma_{3}^{L}+6 \sigma_{4}^{L}+4 \sigma_{5}^{L}+5 \sigma_{6}^{L}, \\
T_{L}\left(1, \sigma^{H}\right)=0 \sigma_{1}^{H}+0 \sigma_{2}^{H}+1 \sigma_{3}^{H}+1 \sigma_{4}^{H}+2 \sigma_{5}^{H}+2 \sigma_{6}^{H}, \\
T_{L}\left(2, \sigma^{H}\right)=0 \sigma_{1}^{H}+0 \sigma_{2}^{H}+0 \sigma_{3}^{H}+2 \sigma_{4}^{H}+2 \sigma_{5}^{H}+3 \sigma_{6}^{H}, \\
T_{L}\left(3, \sigma^{H}\right)=4 \sigma_{1}^{H}+0 \sigma_{2}^{H}+0 \sigma_{3}^{H}+0 \sigma_{4}^{H}+3 \sigma_{5}^{H}+3 \sigma_{6}^{H} .
\end{gathered}
$$

Owing to the symmetricity, only the cases of half locations are listed. Using the argument in the last subsection, firms' location strategy is obtained: $\sigma^{H}=(0,1 / 3,1 / 6,1 / 6,1 / 3,0), \sigma^{L}=(0,1 / 2,0,0,1 / 2,0)$, and their attractions: $T_{H}=5, T_{L}=1$.

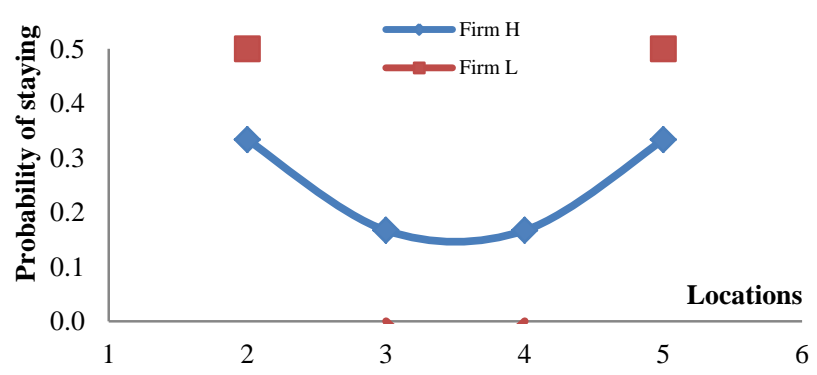

Figure 3. Firms' location strategies when the capability gap is medium

Figure 3 shows that Firm L stays in two niche markets with equal probability and totally gives up center 
positions to focus on these two extremes. The larger capability gap, the more difficult he compete with Firm $\mathrm{H}$. While, Firm $\mathrm{H}$ enjoys more market locations: the central market and the edges. Furthermore, to build up new territories, Firm $\mathrm{H}$ invests more on the edges than on the center. The strategies of Firm $\mathrm{H}$ and Firm L are building and pursuing niches, respectively.

In summary, competing in the same market, firms deploy different location strategies. When the capability gap is none, firms decide to compete back by back, on the market center. When the capability gap is small, weaker firms randomize their location choices. By randomly visiting the market center, the weaker firms force the stronger ones to defend their core market and to leave demands on the boundary. When the capability gap increases, weaker firms pursue niches, most likely investing in the submarkets where the consumers' tastes are special or their demands for quality are high, while stronger firms start to expand their markets, seeking new submarkets or new territories. When the capability gap is sufficiently large, weaker firms will be driven out of market.

\subsection{General Case}

Next to be derived is the most general case where capability gap is small, such as $0<\eta<\frac{1}{m-1}$. Given Firm L's location strategy $\sigma^{L}$, the general form of Equation (3) can be expressed as:

$T_{H}\left(i, \sigma^{L}\right)=\sum_{l=1}^{[(i-1) / 2]}(m-i+l+1)\left(\sigma_{i-2 l-1}^{L}+\sigma_{i-2 l}^{L}\right)+(m-i+1) \sigma_{i-1}^{L}+m \sigma_{i}^{L}+i \sigma_{i+1}^{L}+\sum_{l=1}^{[(m-i) / 2]}(i+l)\left(\sigma_{i+2 l}^{L}+\sigma_{i+2 l+1}^{L}\right)$.

In the choice set of firms' mixed strategies, firms' attractions are the same at any location. Furthermore, the mixed strategy is symmetric along the market center. With these properties, Firm L's optimal strategy can be derived, satisfying

$$
(k-1) \sigma_{k}^{L}=(m-k) \sigma_{K+1}^{L}+\sum_{l=k+2}^{\left[\frac{m}{2}\right]} \sigma_{l}^{L} \quad \text { for } k^{H} \leq k<\left[\frac{m}{2}\right],
$$

as can Firm H's optimal strategy, satisfying:

$$
(k-1) \sigma_{\left[\frac{m}{2}\right]}^{H}=(m-k) \sigma_{\left[\frac{m}{2}\right]-1}^{H}+\sum_{i=k}^{\left[\frac{m}{2}\right]-2} \sigma_{i}^{H} \quad \text { for } k^{L} \leq k<\left[\frac{m}{2}\right],
$$

where $k^{H}$ and $k^{L}$ are the starting locations for Firm H's and Firm L's mixed strategy, respectively. These are summarized in the following proposition.

Proposition 2. When $0<\eta<\frac{1}{m-1}$ and $m>4$, there exists a unique symmetric location strategy for both firms. Mathematically,

1) for Firm L, it is $\left(\sigma_{k^{H}}^{L}, \sigma_{k^{H}+1}^{L}, \ldots, \sigma_{m-k^{H}+1}^{L}\right)$, satisfying $(k-1) \sigma_{k}^{L}=(n-k) \sigma_{k+1}^{L}+\sum_{j=k+2}^{\left[\frac{m}{2}\right]} \sigma_{j}^{L}$ for $k^{H} \leq k<\left[\frac{m}{2}\right]$

2) for Firm $H$, it is $\left(\sigma_{k^{L}}^{H}, \sigma_{k^{L}+1}^{H}, \ldots, \sigma_{m-k^{L}+1}^{H}\right)$, satisfying $(k-1) \sigma_{k}^{H}=(m-k) \sigma_{k+1}^{H}+\sum_{i=k+2}^{\left[\frac{m}{2}\right]} \sigma_{i}^{H}$ for $k^{L} \leq k<\left[\frac{m}{2}\right]$.

Proposition 2 comprises a list of $\left[\frac{m}{2}\right]-k^{L}$ and $\left[\frac{m}{2}\right]-k^{H}$ equations, solvable recursively given the specific value of $m$, as shown in the illustration of Section 3.1. Next, a natural question arises: "What do those mixed strategies of firms look like? Do they show the similar pattern as illustrated in Figure 2?" Proposition 3 states the answer. 
Proposition 3. Firm L's location strategy has a U shape while Firm H's has a reversed U shape. Mathematically, they satisfy the following properties:

1) within the support of Firm L's strategy, $\sigma_{k+1}^{L} \leq \sigma_{k}^{L}$ for $k<\left[\frac{m}{2}\right] ; \sigma_{k+1}^{L} \geq \sigma_{k}^{L}$ for $k>\left[\frac{m}{2}\right]$,

2) within the support of Firm H's strategy, $\sigma_{k+1}^{H} \geq \sigma_{k}^{H}$ for $k<\left[\frac{m}{2}\right] ; \sigma_{k+1}^{H} \leq \sigma_{k}^{H}$ for $k>\left[\frac{m}{2}\right]$, and

3) the starting locations in the support of the mixed strategies satisfies: $k^{H}=k^{L}>\left[\frac{m}{4}\right]$.

(see Appendix B for complete proofs).

Proposition 3 shows the general solution for asymmetric firms to optimally make location choices when their capability gap is small: weaker firms' optimal location strategy takes a U-shape and stronger firms' optimal strategy takes a reversed U-shape, exactly the same as the illustration in Figure 2. Firms' location decisions consider many factors, such as demand, the available market locations, the firm's heterogeneity (strength), and more importantly, the strategic interaction between firms (Alcacer, Dezso, \& Zhao 2013). The difference in their capability induces firms to leverage their competitive position across markets. Weaker firms must differentiate, including randomly visiting the core markets of stronger firms, so stronger firms end up reinforcing their main territories by visiting relatively more on the central market, in a probabilistic sense.

\section{Vertical Differentiation}

In the model outlined so far, consumers are uniformly distributed along the market line. Their locations represent their heterogeneity of their tastes in the features of the project (e.g., content) or product (e.g., colors, styles, shapes, flavors, tastes), and their distances from firms' locations represent the degrees in the mismatch between their tastes and the project/product. The firms' locations cannot be compared. This is the horizontal differentiation location model. In this section, the model is extended to a vertical differentiation location model, where firms' locations can be compared. For example, the location reflects the quality of projects/products (duration, safety, assortment size, speed and service, etc.). Consumers naturally prefer the firm with the project/product of better quality.

Firms. Two asymmetric firms simultaneously make location decisions $\left(x_{n}^{H}, x_{n}^{L}\right)$, where $n$ is the dimension of the project/product and $n \in\{1, \ldots, N\}$. In this case, a consumer's preference is based on the average or combined satisfaction in dimensions, so a firm, even performing lower on certain dimensions, may still win if it performs better on other dimensions. The firm's capability is denoted by $\eta_{j}$, and is normalized within $(0,1] . \quad \eta_{H}>\eta_{L}$. This capability index does not refer directly to the location on the dimension, but rather reflects the efficiency of a firm to conduct business, so that the stronger firm is able to choose a higher sum of locations than the weaker one under same amount of investment. The weaker firm has less leeway to provide greater resources on as many dimensions as the stronger one. A firm's capability index is positively linked to its choice of location as follows:

$$
\frac{x_{1}^{j}+\cdots+x_{N}^{j}}{\eta_{j}}=B
$$

where $B$ is the budget available.

Consumers. Consumers commonly put different weights on dimensions. To keep matters as simple as possible, we start with the simplest case where the weights on dimensions are the same. Let the total market size be normalized to one, then the probability that a consumer prefers Firm $L$ in Dimension $n$, given firms' locations on $\left(x_{i}^{H}, x_{i}^{L}\right)$, is

$$
P_{n}(a \text { consumer prefers Firm } L)=\left\{\begin{array}{cc}
0 & \text { if } x_{n}^{H}>x_{n}^{L} \text { (i.e., Product quality of Firm } H \text { is better on that dimension) } \\
1 / 2 & \text { if } x_{n}^{H}=x_{n}^{L} \text { (i.e., Product quality of both firms are equal on that dimension) } \\
1 & \text { if } x_{n}^{H}<x_{n}^{L} \text { (i.e., Product quality of Firm } L \text { is better on that dimension) }
\end{array}\right.
$$

(9) (Note3)

Analysis. Likewise, consider the cases where capability gaps are small and medium. When the capability gap is zero (i.e., $\eta_{H}=\eta_{L}$ and two firms are symmetric), this model is similar to that of Vandenbosh and Weinberg (1995), who found that firms adopted a MaxMin differentiation strategy: maximize the differentiation of firms' locations in some dimensions but minimize the differentiation in other dimensions. When the capability gap is large, stronger firms outperform weaker ones in all dimensions, thus driving the weak ones out of market. In 
cases where the capability gap is small or medium, with a budget constraint, stronger firms cannot be certain of winning over weak ones in all dimensions, so weak ones stand a chance of taking a bite occasionally by acting strategically.

Firms' mixed strategies are composed of a vector of $N$-univariate marginal distributions, $\left(F_{1}^{j}, \ldots, F_{N}^{j}\right)$. A consumer's preference for a firm, denoted as T, is determined by the firm's average performance over all dimensions. For example, a consumer's preference to Firm L is: $T^{L}=\sum_{n=1}^{N} \frac{P_{n} \text { (consumer prefer Firm L) }}{N}$. Substituting Equation (9) into the above equation, Firm L's decision, which is to maximize consumer's preference under the budget constrain, can be expressed as the following maximization problem:

$$
\begin{aligned}
\max _{\left(F_{1}^{L}, \ldots, F_{N}^{L}\right)} \sum_{n=1}^{N} \int_{0}^{\infty}\left[\frac{1}{N} F_{n}^{H}(x)\right] d F_{n}^{L} \\
\text { S.T. } \quad E\left[x_{1}^{L} \ldots+x_{N}^{L}\right]=B \eta_{L} .
\end{aligned}
$$

Equation (10) is solved by setting up the Lagrangian form for this firm's maximization problem: $L\left(\left(F_{1}^{j}, \ldots, F_{N}^{j}\right), \lambda_{j}\right)=\max _{\left(F_{1}^{j}, \ldots, F_{N}^{j}\right)} \sum_{n=1}^{N} \int_{0}^{\infty}\left[\frac{1}{N} F_{n}^{-j}(x)\right] d F_{n}^{j}+\lambda_{j}\left(B \eta_{j}-E\left[x_{1}^{j} \ldots+x_{N}^{j}\right]\right)$, where $\lambda_{j} \quad$ is $\quad$ the Lagrangian multipliers and $j=\{H, L\}$. Because $\lambda_{j}, B$, and $\eta_{j}$ are constants, the best location strategy for Firm $j$ is equivalent to the solution of the following problem.

$$
L\left(\left(F_{1}^{j}, \ldots, F_{N}^{j}\right), \lambda_{j}\right) \propto \max _{\left(F_{1}^{j}, \ldots, F_{N}^{j}\right)} \sum_{n=1}^{N} \int_{0}^{\infty}\left[\frac{1}{n \lambda_{j}} F_{n}^{-j}(x)-x\right] d F_{n}^{j}
$$

We present the properties of firms' mixed strategies in Lemma 1 to Lemma 4.

Lemma 1. In equilibrium, $\frac{1}{N \lambda_{H}} F_{n}^{L}(x)-x$ is constant for $x$ in the support of Firm H's strategy of $\left(0, s_{H}\right]$; $\frac{1}{N \lambda_{L}} F_{n}^{H}(x)-x$ is constant for $x$ in the support of Firm L's strategy of $\left[0, s_{L}\right]$, where $s_{H}$ and $s_{L}$ are denoted as the boundaries of market coverage for Firm $H$ and Firm L, respectively.

Solving problem (11) is the same as solving the bidding strategy in simultaneous two-bidder all-pay auctions with complete information, as in Bayes, Kovenock and Vries (1996). Lemma 1 is one of their results, so we omit its proof. Note that the upper boundaries of both firms are the same.

Lemma 2. In equilibrium, 1) $\frac{\lambda_{L}}{\lambda_{H}}=\frac{\eta_{H}}{\eta_{L}}$, 2) $s_{i}=\frac{1}{n \lambda_{L}}$, and 3) $\lambda_{L}=\frac{1}{2 \eta_{H} B}$ (see Appendix B for complete proofs).

To test the robustness of the results found previously, a similar analysis applies to the impact of capability gap on location choices under two cases: (1) a small capability gap (i.e., $1 \leq \frac{\eta_{H}}{\eta_{L}}<\frac{N}{2}$ ), and (2) a medium capability gap (i.e., $\left.\frac{N}{2} \leq \frac{\eta_{H}}{\eta_{L}}<(N-1)\right)$.

Next we discussion the firms' location strategies under small and medium capability gaps.

\subsection{Small Capability Gap}

When the capability gap is small, specifically, $1 \leq \frac{\eta_{H}}{\eta_{L}}<\frac{N}{2}$, this condition guarantees that both firms do not exhaust all resources on one dimension. Moreover, limited dimensions are assumed; that is, $8 \geq N \geq 3$. This assumption is the consideration of mind capacity.

Next, of interest is to derive firms' location strategies. Based on Lemma 1, in equilibrium $\frac{1}{N \lambda_{H}} F_{n}^{L}(x)-x$ is 
constant for $x=s_{H}$ and $x$. Therefore, $\frac{1}{N \lambda_{H}} F_{n}^{L}(x)-x=\frac{1}{N \lambda_{H}}-s_{H}$. Further simplified, $F_{n}^{L}(x)=1-s_{H} N \lambda_{H}+$ $x N \lambda_{H}$. After $\lambda_{H}$ and $s_{H}$ in the above equation are substituted by the results from Lemma 2, Firm L's mixed strategy is obtained as follows:

$$
F_{n}^{L}(x)=\left(1-\frac{\eta_{L}}{\eta_{H}}\right)+\left(\frac{\eta_{L}}{\eta_{H}}\right) \frac{x N}{2 \eta_{H^{B}}}, \quad x \in\left(0, \frac{2}{N} B \eta_{H}\right]
$$

Similarly, based on Lemma 1 , in equilibrium $\frac{1}{N \lambda_{B}} F_{n}^{H}(x)-x$ is constant for $x=0$ and $x$. Therefore, $\frac{1}{N \lambda_{B}} F_{n}^{H}(0)-0=\frac{1}{N \lambda_{B}} F_{n}^{H}(x)-x$. Further simplifying, $F_{n}^{H}(x)=x N \lambda_{L}$. Substituting $\lambda_{L}=\frac{1}{2 \eta_{H^{B}}}$ in Lemma 2 into the above equation, Firm H's mixed strategy is obtained as follows:

$$
F_{n}^{H}(x)=\frac{x N}{2 B \eta_{H}}, \quad x \in\left[0, \frac{2}{N} B \eta_{H}\right] .
$$

Firm H's expected attraction is calculated as $T^{H}=\max _{\left(F_{1}^{H}, \ldots, F_{N}^{H}\right)} \sum_{n=1}^{N} \int_{0}^{\infty}\left[\frac{1}{N} F_{n}^{L}(x)\right] d F_{n}^{H}$. Substituting Equation (13) into the above equation yields $T^{H}=\sum_{n=1}^{N} \int_{0}^{\frac{2}{N} B \eta_{H}}\left[\left(1-\frac{\eta_{L}}{\eta_{H}}\right)+\left(\frac{\eta_{L}}{\eta_{H}}\right) \frac{x N}{2 \eta_{H^{B}}}\right] \frac{1}{2 B \eta_{H}} d x$, resulting in

$$
\begin{gathered}
T^{H}=1-\frac{\eta_{L}}{2 \eta_{L}}, \\
T^{L}=1-T^{H}=\frac{\eta_{L}}{2 \eta_{H}} .
\end{gathered}
$$

Under a small capacity gap (i.e. $1 \leq \frac{\eta_{H}}{\eta_{L}}<\frac{N}{2}$ ) firms' mixed strategies are summarized as follows.

Proposition 4. When $1 \leq \frac{\eta_{H}}{\eta_{L}}<\frac{N}{2}$ and $8 \geq N \geq 3$, a unique symmetric location strategy for both firms exists.

Mathematically,

1) for Firm $L, F_{n}^{L}(x)=\left(1-\frac{\eta_{L}}{\eta_{H}}\right)+\left(\frac{\eta_{L}}{\eta_{H}}\right) \frac{x N}{2 \eta_{H} B}, x \in\left(0, \frac{2}{N} B \eta_{H}\right]$;

2) for Firm $H, F_{n}^{H}(x)=\frac{x N}{2 B \eta_{H}}, x \in\left[0, \frac{2}{N} B \eta_{H}\right]$.

Knowing the location strategies in Proposition 4 yields the variation of these location strategies as follows:

$$
\begin{gathered}
\operatorname{var}\left(x_{n}^{L}\right)=\int_{0}^{\frac{2}{N} B \eta_{H}} x^{2} d F_{n}^{L}-\left(\int_{0}^{\frac{2}{N} B \eta_{H}} x d F_{n}^{L}\right)^{2}=\frac{4 B^{2} \eta_{H} \eta_{L}}{3 N^{2}}-\frac{B^{2}\left(\eta_{L}\right)^{2}}{N^{2}} \\
\operatorname{var}\left(x_{n}^{H}\right)=\int_{0}^{\frac{2}{N} B \eta_{H}} x^{2} d F_{n}^{H}-\left(\int_{0}^{\frac{2}{N} B \eta_{H}} x d F_{n}^{H}\right)^{2}=\frac{4 B^{2}\left(\eta_{H}\right)^{2}}{3 N^{2}}-\frac{B^{2}\left(\eta_{H}\right)^{2}}{N^{2}}=\frac{B^{2}\left(\eta_{H}\right)^{2}}{3 N^{2}} .
\end{gathered}
$$

Because $1 \leq \frac{\eta_{H}}{\eta_{L}}<\frac{N}{2}$ and $8 \geq N \geq 3$, comparing Equation (13) with (14) derives:

$$
\operatorname{var}\left(x_{n}^{L}\right)>\operatorname{var}\left(x_{n}^{H}\right)
$$

From Equation (15), a similar nature of Firm L's location strategy is revealed. The firm randomly visits the market locations where Firm H stays, but with less probability, in an attempt to cause Firm H more investment in main markets to protect its dominating positions, leaving the demand at the edge of the markets.

Figure 4 illustrates the location distributions of asymmetric firms as described in Proposition 4. The two firms 
have the same probability of staying on any location in the range of $\left[0, \frac{2}{N} B \eta_{H}\right]$. However, Firm L's distribution has a jump at zero, showing a higher probability to maintain the status quo than at other locations. Moreover, Firm $\mathrm{H}$ is more likely to invest in product renovation.

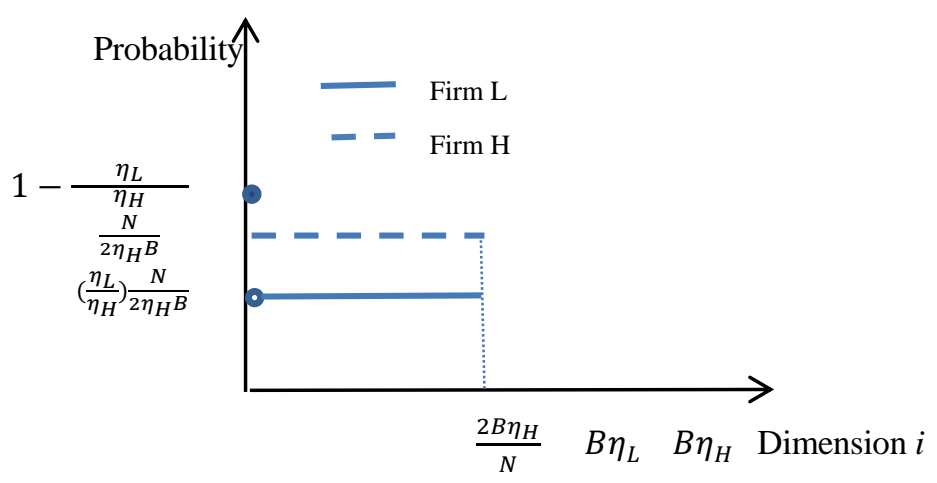

Figure 4. Firms' location strategies when the capability gap is small

\subsection{Medium Capability Gap}

Firms' location strategies also differ under a medium capability gap, specifically, $\frac{N}{2} \leq \frac{\eta_{H}}{\eta_{L}}<(N-1)$, where the weaker firm may focus all of its resources on one dimension.

Proposition 5. When $\frac{N}{2} \leq \frac{\eta_{H}}{\eta_{L}}<(N-1)$ and $8 \geq N \geq 3$, there exists a unique symmetric location strategy for both firms. Mathematically,

1) for Firm $L, F_{n}^{L}(x)=\left(1-\frac{N}{2}\right)+\left(\frac{N}{2}\right) \frac{x}{\eta_{L} B}, x \in\left[\left(1-\frac{2}{N}\right) B \eta_{L}, B \eta_{L}\right]$. Firm L's expected attraction is $\frac{2}{N}-\frac{2}{N^{2}}\left(\frac{\eta_{H}}{\eta_{L}}\right)$

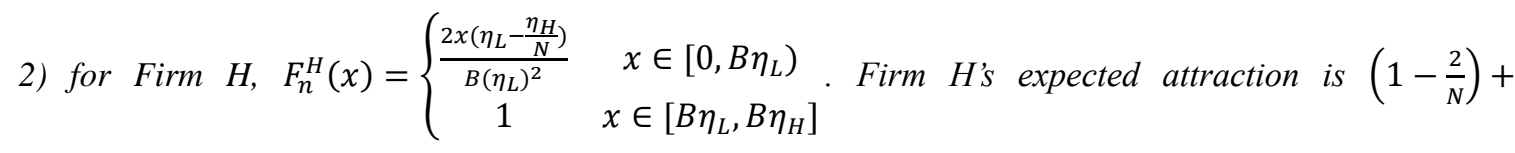
$\frac{2}{N^{2}}\left(\frac{\eta_{H}}{\eta_{L}}\right)$

The proof of Proposition 5 is similar to that for Proposition 4, and thus is omitted. Comparing firms' mixed strategies under different capability gaps reveals that when the capability gap increases, Firm L's attraction decreases and Firm H's increases. For example, Firm L's attraction (i.e., $\frac{2}{N}-\frac{2}{N^{2}}\left(\frac{\eta_{H}}{\eta_{L}}\right)$ ) under a medium capability gap is less than that (i.e., $\frac{\eta_{L}}{2 \eta_{H}}$ ) under a small capability gap. Figure 5 shows the distribution of location strategies. Firm L's location set, $\left[\left(1-\frac{2}{N}\right) B \eta_{L}, B \eta_{L}\right]$, is much smaller, staying on the high end of the dimension. In contrast, Firm H's location set, $\left[0, B \eta_{L}\right]$, is much longer. Intuitively Firm L's optimal strategy is to seek niches, more likely investing in the high quality projects/product. 


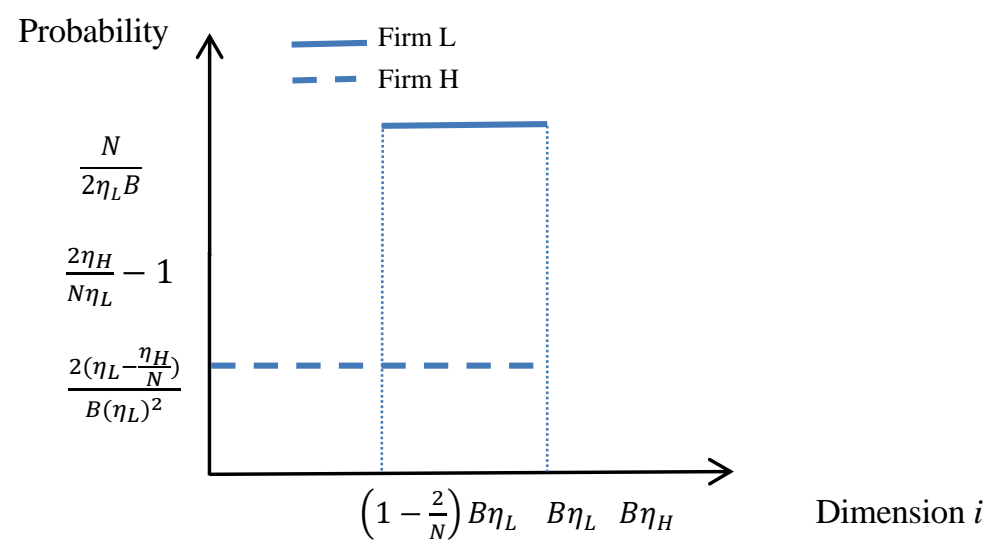

Figure 5. Firms' location strategies when the capability gap is medium

\section{Discussion}

Table 1 summarizes this study's findings of firms' optimal location strategies. Those strategies vary in the nature of the markets and the relative strength of firms (Kotler \& Singh, 1981; Ries \&Trout, 1986). By analogy to military warfare, firms' competitive strategies amount to defending, dodging, building, and pursuing niches to build positions in the chosen marketplace.

Table 1. Firms' location strategies under different levels of capability gap

\begin{tabular}{|c|c|c|c|}
\hline त्ञ్రి & & Firm H's location strategy & Firm L's location strategy \\
\hline 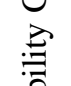 & Small & $\begin{array}{c}\text { Defend } \\
\text { (Focus on core markets) }\end{array}$ & $\begin{array}{c}\text { Dodge } \\
\text { (Randomize) }\end{array}$ \\
\hline 㱒 & Medium & $\begin{array}{c}\text { Build } \\
\text { (Extend market places) }\end{array}$ & $\begin{array}{c}\text { Pursue niches } \\
\text { (Focus on niche markets) }\end{array}$ \\
\hline
\end{tabular}

Firm H's location strategy turns from "focusing on core competencies" to "expanding aggressively to the edges" and from "investing in the strength" to "compensating for the weakness" after noticing its rival has retreated from the mass market. The strategy adopted by a stronger firm is either defending or building: (1) Defend. When the capability gap is small, a firm already strong in the market may pursue an essentially defensive strategy to enable it to hold and win the current position against its potential attackers, especially when its product is mature. (2) Build. When the capability gap is medium, a firm already strong in the market may turn to a building strategy, aggressively expanding its domestic and/or international markets and taking sales from competitors.

In contrast, Firm L's location strategy turns from "randomization" to "focusing" and from "gorilla attack" to "fighting for survival." The level of randomization is negatively related with the capability gap. The strategy adopted by a weaker firm is either dodging or pursuing niches.

Dodge. When a firm finds itself less competitive in the market, a common reaction is to go to a market niche to get a larger share of a smaller market rather than compete with a stronger firm to get a smaller share of a large market. However, this study shows that simply choosing to stay in niches is not optimal when the capability gap is small. Instead, a weaker firm should randomly step into the core markets of its rival. By doing so, it is able to generate threats to its rival so that the competitor has to defend core markets, thus leaving large shares in market niches to the weaker firm. For example, Thomadsen (2007) checked the geographical location choices of McDonald's and Burger King outlets in areas of Santa Clara. Along a stretch of El Camino Real, Thomadsen found a random pattern on the nearest distances between two sellers' outlets: in some areas, the distance was very close and in other areas, the distance was very far. Another manifestation of the dodging strategy is the rising popularity of "guerrilla marketing" as an advertising strategy for online social media. Firms avoid the markets where the competition is high and instead carry out eye-catching and surprising marketing activities, thus being highly efficient in terms of gaining customer attention - a "hit and run" tactic.

Pursue Niches. When the relative strength is medium, a weaker firm should resist the temptation of reaching all consumers or imitating its competitors and instead fine-tune its projects and products to the particular and often unique needs of customers. Having projects/products that differ substantially from those of other firms can avoid direct competition. More importantly, by focusing on a narrower sector of the total market, the firm is able to concentrate its resources and become specialized. By providing a special touch to consumers, the firm is able to compensate for some of the disadvantage of small size. 
The footwear industry offers an example. This market is dominated by three giants, Nike, Adidas, and Puma, and also has many smaller players such as Lululemon, Under Armour, Asics, Mizuno, Brooks, and others. Puma is currently third in the sporting-goods industry, a slightly weaker than Nike and Adidas. Although it covers target markets similar to those of the other two giants, it positions itself as a desirable lifestyle brand, concentrating more on fashion lifestyles. And a much smaller company like Saucony further narrows its target markets on running shoes.

This study finds that although larger firms invest more in research than smaller firms, smaller firms are more likely to invest in "radical" innovations (Kanter, 1985; Chakrabarti \& Halperin, 1990; Salavou \& Lioukas, 2003). Small firms provide considerably more innovation per research dollar than do the large firms (Tornatzsky, 1983). For example, when major automobile manufacturers concentrate their attention on large-scale production and standardization of product parts to enjoy the economies of scale of assembly lines, many smaller firms focus on small markets, such as the market for high-quality and hand-crafted cars (Guerzoni, 2014). Recently Google entered the automobile industry, investing in the R\&D of self-driving cars. To avoid competing with automotive giants, Google aims to reach a small group of consumers who are not fond of driving - a small niche.

\section{Limitations and Future Research}

This study models the optimal location strategy for a weaker firm when competing with a strong one. However, to maintain simplicity of the model, some important dimensions of location choices have been left out. The results should be viewed in light of the model's assumptions. The following discussion examines in details the relaxation of some assumptions, such as about consumers' distribution, firm capability, and non-price competition.

\subsection{Consumers' Distribution}

In this paper, consumers are assumed to be discretely distributed in the marketplace of $[0,1]$. Such an assumption allows the use of simple examples to demonstrate the location choices. An alternative assumption on consumers' location is a continuous distribution-suppose consumers are continuously and uniformly distributed in the marketplace of $[0,1]$. As in Proposition 1, the non-existence of pure strategies is summarized as follows.

Proposition 6. When the capability between firms is small or medium, pure location strategies for the firms do not exist (see Appendix B for complete proofs).

The next is to examine the firms' mixed strategies. Let market coverage in the firms' mixed strategies be $[\underline{x}, \bar{x}]$. $\bar{x}=1-\underline{x}$, owing to the symmetry of strategies. Let Firm H stay on the left of Firm L and $x$ be a consumer's location where she is indifferent to purchasing from Firm H or Firm L. Then that consumer's utility function satisfies:

$$
\begin{gathered}
t_{0}+t-\left(x-x_{H}\right)^{2}=t_{0}-\left(x-x_{L}\right)^{2} . \\
\text { Then } x=\frac{x_{L}^{2}+t-x_{H}{ }^{2}}{2\left(x_{L}-x_{H}\right)}, \\
T_{H}=x, \text { and } T_{L}=1-x .
\end{gathered}
$$

The two firms' attractions when $x_{H}=\underline{x}$ or $\frac{1}{2}$, and $x_{L}=\underline{x}$ or $\frac{1}{2}$ are as follows:

$$
\begin{aligned}
& T_{H}\left(x_{H}=\underline{x}, \sigma^{L}\right)=\int_{\underline{x}}^{\bar{x}} \frac{x_{L}^{2}+t-\underline{x}^{2}}{2\left(x_{L}-\underline{x}\right)} \sigma^{L} d x_{L}, \\
& T_{H}\left(x_{H}=\frac{1}{2}, \sigma^{L}\right)=\int_{\underline{x}}^{\frac{1}{2}} \frac{x_{L}{ }^{2}+t-\frac{1^{2}}{2}}{2\left(x_{L}-\frac{1}{2}\right)} \sigma^{L} d x_{L}+\int_{\frac{1}{2}}^{\bar{x}}\left(1-\frac{x_{L}{ }^{2}+t-\frac{1^{2}}{2}}{2\left(x_{L}-\frac{1}{2}\right)}\right) \sigma^{L} d x_{L} \text {, } \\
& T_{L}\left(\sigma^{H}, x_{L}=\underline{x}\right)=\int_{\underline{x}}^{\bar{x}}\left[1-\frac{\underline{x}^{2}+t-x_{H}{ }^{2}}{2\left(\underline{x}-x_{H}\right)}\right] \sigma^{H} d x_{H} \text {, and } \\
& T_{L}\left(\sigma^{H}, x_{L}=\frac{1}{2}\right)=\int_{\underline{x}}^{\frac{1}{2}}\left[1-\frac{\left(\frac{1}{2}\right)^{2}+t-x_{H}^{2}}{2\left(\frac{1}{2}-x_{H}\right)}\right] \sigma^{H} d x_{H}+\int_{\frac{1}{2}}^{\bar{x}} \frac{\left(\frac{1}{2}\right)^{2}+t-x_{H}{ }^{2}}{2\left(\frac{1}{2}-x_{H}\right)} \sigma^{H} d x_{H} .
\end{aligned}
$$

A mixed strategy consists of a pair of probability distributions over the respective strategy space with the property that for each player's mixed strategy, any actions chosen with positive probability must be optimal against the other player's reaction and equal. Thus, 


$$
\begin{aligned}
& \int_{\underline{x}}^{\bar{x}} \frac{x_{L}^{2}+t-\underline{x}^{2}}{2\left(x_{L}-\underline{x}\right)} \sigma^{L} d x_{L}=\int_{\underline{x}}^{\frac{1}{2}} \frac{x_{L}^{2}+t-\frac{1^{2}}{2}}{2\left(x_{L}-\frac{1}{2}\right)} \sigma^{L} d x_{L}+\int_{\frac{1}{2}}^{\bar{x}}\left(1-\frac{x_{L}^{2}+t-\frac{1}{2}}{2\left(x_{L}-\frac{1}{2}\right)}\right) \sigma^{L} d x_{L},
\end{aligned}
$$

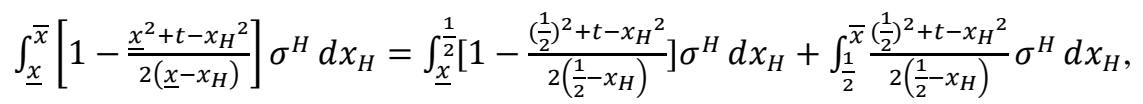

The optimal strategies $\left(\sigma^{H}, \sigma^{L}\right)$ satisfy the conditions in Equations (16) and (17). The complexity of the equations forbids deriving clean solutions. However, the mixed strategies exist because the game is compact, Hausdorff, zero sum (i.e., the game is reciprocally semi-continuous), and payoff secure (Note 4), satisfying the existing conditions of mixed strategy (Reny, 1999; Carmona, 2009).

\subsection{Firms' Capability}

Firms' capability refers to the firm's ability to execute its business strategy. Other alternatives exist besides the approach modeled in the paper. First, it may be modeled from the firm's view, allowing for different costs in product development, operation, or financial resources. In this case, it is shown in firms' profit functions. Second, it may be modeled from the consumer's view, allowing for firms' differing ability to shorten the distance from consumers' ideal locations and differing brand equity, services or convenience provided (such as shorter shipping time, or the possibility a consumer can rent a car in a given city and return it at any location worldwide) and so on. Then capability is shown as an additional parameter added into consumers' utility functions. Third, it may be modeled from market share (Seyman Hoch, \& Raju, 2002; Budd, Harris, \& Vickers, 1993). Then it is shown in the demand function. Further research should consider alternative approaches to model firms' capability.

\subsection{Price}

The paper does not consider price as one of the decision variables - a case that would fit a number of situations. These situations include (1) R\&D of a core technique to be applied to a series of products, where the firm may make the location decisions first before setting prices of specific products; (2) decisions in the communication industry of television, radio, newspapers, magazines, and direct mail, where positioning strategies do not involve price competition; and (3) decisions in the markets of agriculture products, where the prices are determined not by a single firm but by total demands and supplies. For example, Rust and Donthu (1988) analyzed the positioning and repositioning of cable networks without the consideration of prices. They observed that, given that a perceptual space exists for viewers, all movie channels (HBO, SHOWTIME, and CINEMAX) were perceived to be similar and hence were located near each other. ESPN and CNN were located in two different and relatively isolated areas owing to their unique programming contents, while LIFETIME, USA, and WTBS had broad programming and did not focus on any one type of show and were perceived to be at the center, without a unique target audience. Therefore, new cable channels should adopt locations that were highly associated with investigative news reporting programs or action/mystery shows.

However, in many more cases price is a major consideration. Numerous analytical models have considered pricing competition when analyzing product positioning, modeling the problem as a two-stage sequential game in which firms decide their product positioning first and then make price decisions (D'Aspremont, Gabszewicz, $\&$ Thisse 1979).

An additional extension of the model is that consumers put different weights on dimensions in the vertical differentiation location model. For example, when evaluating a laptop, consumers may consider battery life, speed, and weight to be the top features, while the aesthetics, service and color may be less important. Further research could consider the model with varied consumers' weights on dimensions.

\section{Conclusion}

This investigation is a starting point for understanding how firms' capability gap shapes their location strategies. As most theoretical location studies have focused on symmetric firms or entry models, rigorous theoretical analyses on asymmetric firms are still missing. This paper uses simple game-theoretical models to provide a theoretical explanation for why weaker firms sometimes differentiate their positioning (i.e., dodge) and sometimes focus on narrower submarkets (i.e., pursue niches) and why weaker firms are more likely to develop radical renovations.

By better understanding the relationship between firms' capability gap and their strategic interactions, managers can determine what their strategies should be in the evolution of the industry. Stronger firms may prosper because of their strong reputation, quality products and services, and superior distribution. However, smaller firms may make a move that changes the industry fundamentally, for example, by applying radical technologies 
which provide high profits.

A strong marketing strategy, such as an advertising campaign or investment in $R \& D$, may lead to the perception that strong/large firms contribute more to innovation and radical invention. This study reveals the opposite: smaller/weaker firms are the sources of most "radical products." This finding is supported by the flush of radical innovations in Silicon Valley in a highly competitive high-technology industry. Under intense competition, weaker/smaller firms increase their investment in radical or fundamental products for survival, or alter/achieve a new form of competitive advantage.

The paper provides a strategic guide for marketing managers on positioning and resource allocation. Further, the insights offered by the paper contribute to the theory of marketing strategy of asymmetric firms, especially small- and medium-sized firms. As over $99.9 \%$ of employer firms are SMEs, generating over $50 \%$ of employment, our study holds huge benefits, not only for small- and medium-sized business, but also for national economic growth and human welfare.

\section{References}

Alcácer, J., Dezső, C., \& Zhao, M. (2013). Firm rivalry, knowledge accumulation, and MNE location choices. Journal of International Business Studies, 44(5), 504-520. https://doi.org/10.1057/jibs.2013.18

Ansolabehere, S., Snyder, J., \& Stewart, C. (2001). Candidate positioning in U.S. house elections. American Journal of Political Science, 45(1), 136-159. https://doi.org/10.2307/2669364

Baye, M., Kovenock, D., \& De Vries, C. (1996). The all-pay auction with complete information. Economic Theory, 8(2), 291-305. https://doi.org/10.1007/BF01211819

Berry, S. (1992). Estimation of a model of entry in the airline industry. Econometrica, 60(4), 889-917. https://doi.org/10.2307/2951571

Berry, S. (1994). Estimating Discrete-Choice Models of Product Differentiation. The RAND Journal of Economics, 25(2), 242-262. https://doi.org/10.2307/2555829

Borel, E., \& Ville, J. (1938). Application de la theorie des Probabilites aux jeux de hazard. Gauthier-Villars, Paris.

Budd, C., Harris, C., \& Vickers, J. (1993). A model of the evolution of duopoly: Does the asymmetry between firms tend to increase or decrease? The Review of Economic Studies, 60(3), 543-573. https://doi.org/10.2307/2298124

Carmona, G. (2009). An existence result for discontinuous games. Journal of Economic Theory, 144, 1333-1340. https://doi.org/10.1016/j.jet.2008.11.004

Carpenter, G., \& Nakamoto, K. (1990). Competitive strategies for late entry into a market with a dominant brand. Management Science, 36(10), 1268-1278. https://doi.org/10.1287/mnsc.36.10.1268

Chakrabarti, A., \& Halperin, M. (1990). Technical performance and firm Size: Analysis of patents and publications of U.S. firms. Small Business Economics, 2(3), 183-190. https://doi.org/10.1007/BF00389526

D'Aspremont, C., Gabszewicz, J., \& J. F. Thisse. (1979). On Hotelling's "stability in competition". Econometrica, 47(5), 1145-1150. https://doi.org/10.2307/1911955

De Palma, A., Ginsburgh, V., Papageorgiou, Y., \& J. F. Thisse. (1985). The principle of minimum differentiation holds under sufficient heterogeneity. Econometrica, 53(4), 767-781. https://doi.org/10.2307/1912653

Du, R., Lee, E., \& Staelin, R. (2005). Bridge, focus, attack, or stimulate: retail category management strategies with a store brand. Quantitative Marketing and Economics, 3(4), 393-418. https://doi.org/10.1007/s11129-005-2779-8

Economides, N. (1986). Nash equilibrium in duopoly with products defined by two characteristics. The RAND Journal of Economics, 17(3), 431-439. https://doi.org/10.2307/2555722

Friedman, L. (1958). Game-theory models in the allocation of advertising expenditures. Operations Research, 6(5), 699-709. http://www.jstor.org/stable/166896

Groseclose, T. (2001). A model of candidate location when one candidate has a valence advantage. American Journal of Political Science, 45(4), 862-886. https://doi.org/10.2307/2669329

Gross, O., \& Wagner, R. (1950). A continuous Colonel Blotto game. RAND Corporation, https://www.rand.org/pubs/research_memoranda/RM408.html 
Guerzoni, M. (2014). Product variety in automotive industry: understanding niche markets in America, Springer Science \& Business Media. https://doi.org/10.1007/978-3-319-01907-9

Hotelling, H. (1929). Stability in competition. The Economic Journal, 39(153), 41-57. https://doi.org/10.2307/2224214

Kanter, R. (1985). Supporting innovation and venture development in established companies. Journal of Business Venturing, 1(1), 47-60. https://doi.org/10.1016/0883-9026(85)90006-0

Kobe, K. (2012). Small business GDP: Update 2002-2010. Economic Consulting Services, LLC, Washington, DC.

Kotler, P., \& Singh, R. (1981). Marketing warfare in the 1980s. Journal of Business Strategy, 1, 30-41.

Krasa, S., \& Polborn, M. (2010). Competition between specialized candidates. The American Political Science Review, 104(4), 745-765. https://doi.org/10.1017/S0003055410000468

Link, A., \& Bozeman, B. (1991). Innovative behavior in small-sized firms. Small Business Economics, 3(3), 179-184. https://doi.org/10.1007/BF00400023

Mazzeo, M. (2002). Product choice and oligopoly market structure. The RAND Journal of Economics, 33(2), 221-242. https://doi.org/10.2307/3087431

Moorthy, K. (1988). Product and price competition in a duopoly. Marketing Science, 7(2), 141-168. https://doi.org/10.1287/mksc.7.2.141

Netz, J., \& Taylor, B. (2002). Maximum or minimum differentiation? Location patterns of retail outlets. The Review of Economics and Statistics, 84(1), 162-175. https://doi.org/10.1162/003465302317331991

Porter, M. (1980). Competitive strategy. New York: The Free Press.

Reny, P. (1999). On the existence of pure and mixed strategy Nash equilibria in discontinuous games. Econometrica, 67(5), 1029-1056. https://doi.org/10.1111/1468-0262.00069

Ries, A., \& Trout, J. (1986). Marketing warfare. Journal of Consumer Marketing, 3(4), 77-82. https://doi.org/10.1108/eb008182

Roberson, B. (2006). The Colonel Blotto game. Economic Theory, 29(1), 1-24. https://doi.org/10.1007/s00199-005-0071-5

Salavou, H., \& Lioukas, S. (2003). Radical product innovations in SMEs: the dominance of entrepreneurial orientation. Creativity and Innovation Management, 12(2), 94-108. https://doi.org/10.1111/1467-8691.00272

Salop, S. (1979). Monopolistic competition with outside goods. The Bell Journal of Economics, 10(1), 141-156. https://doi.org/10.2307/3003323

Seim, K. (2006). An empirical model of firm entry with endogenous product-type choices. The RAND Journal of Economics, 37(3), 619-640. https://doi.org/10.1111/j.1756-2171.2006.tb00034.x

Seyman, S., Hoch, S., \& Raju, J. (2002). Positioning of store brands. Marketing Science, 21(4), 278-397. https://doi.org/10.1287/mksc.21.4.378.134

Szentes, B., Rosenthal, R. (2003). Three-object, two-bidder simultaneous auctions: Chopsticks and tetrahedral. Games and Economic Behavior, 44, 114-133. https://doi.org/10.1016/S0899-8256(02)00530-4

Thomadsen, R. (2007). Product Positioning and Competition: The Role of Location in the Fast Food Industry. Marketing Science, 26(6), 792-804. https://doi.org/10.1287/mksc.1070.0296

Thomas, L., \& Weigelt, K. (2000). Product location choice and firm capabilities: Evidence from the U.S. Automobile Industry. Strategic Management Journal, 21(9), 897-909. https://doi.org/10.1002/1097-0266(200009)21:9<897::AID-SMJ140>3.0.CO;2-X

Tirole, J. (1988). The theory of industrial organization. MIT Press

Tornatzsky, L. (1983). The process of technological innovation: reviewing the literature. Washington, D.C.: National Science Foundation (May).

Trout, J., \& Ries, A. (1978). Recycling battles: study the classics to avoid checkmate in business war. Marketing Times, 25(May/June), 17-20.

Tyagi, R. (2000). Sequential product positioning under differential costs. Management Science, 46(7), 928-940. 
https://doi.org/10.1287/mnsc.46.7.928.12038

Vandenbosch, M., \& Weinberg, C. (1995). Product and price competition in a two-dimensional vertical differentiation model. Marketing Science, 14(2), 224-249. https://doi.org/10.1287/mksc.14.2.224

Watson, R. (2009). Product variety and competition in the retail market for eyeglasses. The Journal of Industrial Economics, 57(2), 217-251. https://doi.org/10.1111/j.1467-6451.2009.00381.x

\section{Notes}

Note 1. In the equation, "[x]" denotes the smallest integer larger than or equal to $\mathrm{x}$. For subscripts that fall outside the range, the values of those terms are set to 0 .

Note 2. In the illustration, $\mathrm{m}$ is chosen to be even for the convenience. The nature of result also holds when $\mathrm{m}$ is odd. Especially when $\mathrm{m}$ is greater, the mixed strategies when $\mathrm{m}$ is odd and when $\mathrm{m}$ is even converge.

Note 3. More than one way exists to model the probability of a firm winning over a consumer, for example, $P_{n}($ conumers prefer Firm L $)=\frac{x_{n}^{L}}{x_{n}^{L}+x_{n}^{H}}$.

Note 4. Reny (1999, p. 1030) defines: "A game is payoff secure if for every joint strategy, x, each player has a strategy that virtually guarantees the payoff he receives at $\mathrm{x}$, even if the others play slightly differently than at x."

\section{Appendix A}

\section{A Colonel Blotto game}

The battle field has two sites. Colonel Blotto owns 4 regiments of troops and the opposing commander, Colonel Lotso, owns 3 regiments. In the field, the army with more troops in the site wins the site. If one Colonel sends $\boldsymbol{x}$ troops and the other sends y with $y<x$, the Colonel who sent $\boldsymbol{x}$ wins and obtains a payoff of $\boldsymbol{y}+1$, where $\mathrm{y}$ is the number of troops captured, and 1 is a bonus for securing the site. If they send all their troops to opposite sites, although each securing one site, they don't defeat any enemy. Therefore, each gets a payoff of 0 . How should the two Colonels deploy their troops to win the battle?

Analysis. Colonel Blotto has five possible decisions: $(4,0),(0,4),(3,1),(1,3)$, and $(2,2)$. The first number refers to the troops sent to the first site, and the second the troops sent to the second site. For example, $(4,0)$ means that Colonel Blotto sends all his troops to the first site and zero to the second. Similarly, Colonel Lotso has four possible decisions: $(3,0),(0,3),(2,1)$, and $(1,2)$. Therefore, a 5 x 4 matrix is used to hold payoffs under different combinations of their decisions (See Table 2).

Table 1. Two Colonels' payoffs under different combinations of their decisions

\section{Lotso}

\begin{tabular}{rl|c|c|c|c|} 
& Payoff & \multicolumn{1}{c}{$(3,0)$} & \multicolumn{1}{c}{$(0,3)$} & \multicolumn{1}{c}{$(2,1)$} & \multicolumn{1}{c}{$(1,2)$} \\
\cline { 3 - 6 } & $(4,0)$ & $(4,-4)$ & $(0,0)$ & $(2,-2)$ & $(1,-1)$ \\
\cline { 3 - 6 } & $(0,4)$ & $(0,0)$ & $(4,-4)$ & $(1,-1)$ & $(2,-2)$ \\
\cline { 3 - 6 } & $(3,1)$ & $(1,-1)$ & $(-1,1)$ & $(3,-3)$ & $(0,0)$ \\
\cline { 3 - 6 } & $(-1,1)$ & $(1,-1)$ & $(0,0)$ & $(3,-3)$ \\
\cline { 3 - 6 } & $(1,3)$ & $(-1,1)$ & $(2,-2)$ & $(2,-2)$ \\
\cline { 3 - 6 } & $(2,2)$ & $(-2,2)$ & $(-2,2)$ &
\end{tabular}

In the payoff cell, the first number refers to Colonel Blotto's payoff, and the second to Colonel Lotso's. For example, $(1,-1)$ is the payoff of the battle when Blotto adopted the strategy of $(4,0)$ and Lotso adopted the strategy of $(1,2)$. In the battle, Blotto wins the first site, but loses the second. His payoff thus equals 1 (capture one troop of his enemy $)+1$ (secure the first site $)-1$ (lose the second site $)=1$. Lotso loses in the first site but wins in the second, so his payoff equals -1(lose one troop) -1 (lose the first site) +1 (win the second site) $=-1$. The sum of both Colonels' payoffs is zero, a zero-sum game.

The next is to find out the two colonels' optimal strategies. Let Blotto's mixed strategy be denoted by $p, p, q, q$, 1-2p-2q, the probabilities of choosing decisions listed in the first column. Let Lotso's mixed strategy be denoted by $r, r, 1 / 2-r, 1 / 2-r$, the probabilities of choosing decisions listed in the first row. Then Blotto's payoffs of choosing 
the decisions in the first column are: for Row 1: $4 r+0+2(1 / 2-r)+(1 / 2-r)$, which is simplified as $3 / 2+$ $r$; for Row 2: the same as Row 1, owing to the symmetry between Row 1 and Row 2; for Row 3: $r-r+3(1 / 2-$ $r)+0$, which is simplified as $3 / 2-3 r$; for Row 4: the same as Row 3, owing to the symmetry between Row 3 and Row 4; for Row 5: $-4 r+4(1 / 2-r)$, which is simplified as $2-8 r$. In equilibrium, Blotto's payoffs for rows are equal, yielding $r=0$, or $r=1 / 18$.

Lotso's payoffs of choosing decisions listed in the first row are: for Column 1: $-4 p+0-q+q+2(1-2 p-2 q$ ), which is simplified as $2-8 p-4 q$; for Column 2: the same as Column 1, owing to the symmetry between Column 1 and Column 2; for Column 3: $-2 p-p-3 q-0-2(1-2 p-2 q)$, which is simplified as $-2+p+q$; for Column 4: the same as Column 3, owing to the symmetry between Column 3 and Column 4. In equilibrium, Lotso's payoffs for columns are equal, yielding $9 p+5 q=4$.

The next step is to show that $r=0$ is not a solution. Suppose it is. Then Lotso adopts the last two decisions with equal probability of 50 (so the first two columns are crossed-out). Knowing this, Blotto will choose the last decision, because the four payoffs are dominated by that of the last decision (the first four rows are crossed-out). Deleting unused decisions yields a much smaller matrix as Table 3:

Table 2. Eliminating dominated strategies in two Colonels' payoff matrix

\section{Lotso}

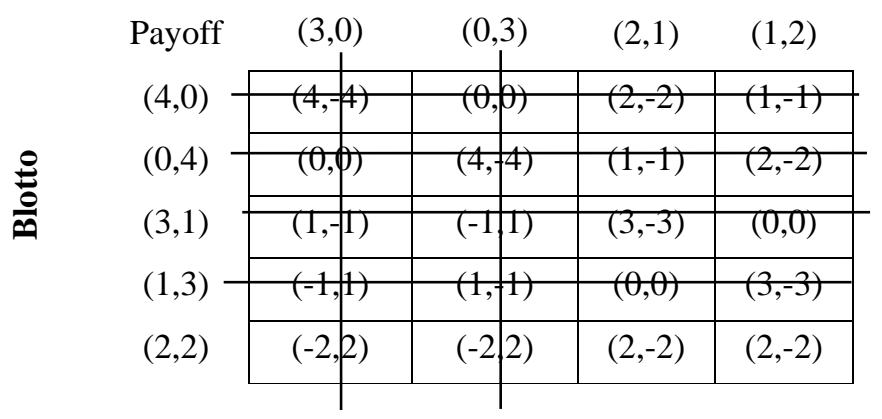

In the above new matrix, Lotso will deviate because his payoff is much lower than when he chooses other decisions. Therefore, $r=0$ is not an equilibrium solution. Then Lotso's mixed strategy is $1 / 18,1 / 18,7 / 18,7 / 18$. Blotto's payoffs under different decisions are: for Row 1:3/2 $+r=14 / 9$; for Row 2:14/9; for Row 3: $3 / 2-3 r=$ 12/9; for Row 4: 12/9; for Row 5: $2-8 r=14 / 9$.

From the above, the payoffs of Blotto's decisions for Row 3 and Row 4 are dominated by those of the decisions for other rows; therefore $q=0$. With the knowledge of $9 p+5 q=4$, which is derived earlier, $p=4 / 9$ is derived. Two Colonels' mixed strategies and payoffs are summarized in Table 4.

Table 4. Mixed strategies and payoffs of two Colonels' mixed strategies and payoffs

$\begin{array}{ccccccc}\text { Blotto } & \underline{\text { Decision set }} & \underline{(4,0)} & \underline{(0,4)} & \underline{(3,1)} & \underline{(1,3)} & \underline{(2,2)} \\ & \text { Mixed strategy } & 4 / 9 & 4 / 9 & 0 & 0 & 1 / 9 \\ & \text { Payoff } & 14 / 9 & & & & \\ \text { Lotso } & \underline{\text { Decision set }} & \underline{(3,0)} & \underline{(0,3)} & \underline{(2,1)} & \underline{(1,2)} & \\ & \text { Mixed strategy } & 1 / 18 & 1 / 18 & 4 / 9 & 4 / 9 & \\ & \text { Payoff } & -4 / 9 & & & & \end{array}$

Interpretation. When both colonels are playing optimally in the battle field, Colonel Blotto is expected to gain 14/9, while Colonel Lotso is expected to lose 14/9, owing to Colonel Blotto's one extra regiment. As the capability gap is still small, Colonel Blotto should concentrate his troops to specific sites and occasionally split his troops, just to make sure that Lotso cannot steal a location easily. In contrast, Colonel Lotso is not able to win against Blotto in the numbers, so he has to spread his troops out and hopes to secure an undefended location with one regiment. Occasionally Colonel Lotso should deploy all his troops to one site or the other, to make Colonel Blotto unable to spread his troops evenly all the time. 


\section{Appendix B}

\section{Proof of Proposition 1}

Suppose that firms' pure strategy exists. Let the pure strategy denoted by $\left(x_{H^{*}}, x_{L^{*}}\right)$ for Firm $\mathrm{H}$ and L, respectively. The basic idea of pure strategy is that if one player chooses this strategy, then no other player has incentives to deviate from that strategy. Furthermore, $\left(x_{H^{*}}, x_{L *}\right)$ is a fixed point, thus predictable by both firms before they make their location decisions.

1) If two points are at the same location - that is, $x_{H^{*}}=x_{L^{*}}$, then Firm $\mathrm{H}$ gets all consumers, owing to his competitive advantage. However, if Firm L moves away from the current position, he can obtain some positive market share when the capability gap is small or medium. Firm $L$ has an incentive to deviate from $x_{L^{*}}$, so $\left(x_{H *}, x_{L *}\right)$ is not an equilibrium solution.

2) If two points are different- that is, $x_{H^{*}} \neq x_{L^{*}}$, then Firm $\mathrm{H}$ has the incentive to move to $x_{L^{*}}$. By doing so, he can capture all consumers and becomes more profitable. Therefore $\left(x_{H^{*}}, x_{L_{*}}\right)$ is not an equilibrium solution, either.

In summary, the above two cases show that at least one firm has an incentive to deviate from $\left(x_{H^{*}}, x_{L^{*}}\right)$.

Therefore no pure strategy exists.

\section{Proof of Proposition 3}

1) $k<\frac{m}{2}$ means that $k<\frac{m+1}{2}$, and then $k-1<m-k$.

$$
k-1<m-k \Rightarrow(k-1) \sigma_{k}^{L}<(m-k) \sigma_{k}^{L}
$$

From (6), $(k-1) \sigma_{k}^{L}=(m-k) \sigma_{k+1}^{L}+\sum_{i=k+2}^{\frac{n}{2}} \sigma_{i}^{L}$ is derived for $k<\frac{m}{2}$. Substituting $(k-1) \sigma_{k}^{L}$ in the above equation into to $(k-1) \sigma_{k}^{L}<(m-k) \sigma_{k}^{L}$ yields the following:

$$
(m-k) \sigma_{k+1}^{L}+\sum_{i=k+2}^{m / 2} \sigma_{i}^{L}<(m-k) \sigma_{k}^{L}
$$

For this inequality to be held, $\sigma_{k+1}^{L} \leq \sigma_{k}^{L}$ for $k<\frac{m}{2}$.

The second part can be proved in the same way.

2) Part 2 can be proved in the similar way.

3) Suppose the opposite - when $k \leq \frac{m}{4}, \sigma_{k}^{H}>0$. Then $\leq \frac{m}{4} \Rightarrow 2 k \leq \frac{m}{2} \Rightarrow k-1 \leq \frac{m}{2}-k-1 \Rightarrow$ $(k-1) \sigma_{k}^{H} \leq\left(\frac{m}{2}-k-1\right) \sigma_{k}^{H}=\sum_{i=k+2}^{\frac{m}{2}} \sigma_{k}^{H}$.

According to Proposition 2, $\sigma_{k+1}^{H}>\sigma_{k}^{H}$, yielding $(k-1) \sigma_{k}^{H} \leq \sum_{i=k+2}^{\frac{m}{2}} \sigma_{i}^{H}$.

In Equation (7), Firm H's strategy is $(k-1) \sigma_{k}^{H}=(m-k) \sigma_{k+1}^{H}+\sum_{i=k+2}^{\frac{m}{2}} \sigma_{i}^{H}$. Submitting Equation (7) to the above inequality yields $\sigma_{k}^{H}=0$ for $k \leq \frac{m}{4}$, which is contradictory to what is supposed at the beginning (i.e., when $\left.k \leq \frac{m}{4}, \sigma_{k}^{H}>0\right)$. 
From (6), $k^{H} \leq k^{L}$, because $k^{L}$ shall be in the support of Firm L's strategy. From (7), $k^{L} \leq k^{H}$, because $k^{H}$ shall be in the support of Firm H's strategy. Therefore, $k^{H}=k^{L}$.

\section{Proof of Lemma 2}

Part 1: Because each firm uses up all the resources, then $\mathrm{E}\left[\frac{x_{1}^{j} \ldots+x_{N}^{j}}{\eta_{j}}\right]=B$.

$$
\begin{gathered}
\Rightarrow \quad \frac{E\left[x_{1}^{H} \ldots+x_{N}^{H}\right\}}{\eta_{H}}=\frac{E\left[x_{1}^{L} \ldots+x_{N}^{L}\right]}{\eta_{L}}, \\
\Rightarrow \quad \frac{\sum_{n=1}^{N} \int_{0}^{\infty} x d F_{n}^{H}(x)}{\eta_{H}}=\frac{\left.\sum_{n=1}^{N} \int_{0}^{\infty} x d F_{n}^{L}(x)\right]}{\eta_{L}} .
\end{gathered}
$$

Lemma 1 shows that $\frac{1}{n \lambda_{L}} F_{n}^{H}(x)-x=$ constant, which means

$$
d F_{n}^{-j}(x)=N \lambda_{j} d x
$$

Substituting (B-2) to (B-1) yields

$$
\begin{aligned}
\frac{\sum_{n=1}^{N} \int_{0}^{\infty} x n \lambda_{L} d x}{\eta_{H}} & =\frac{\sum_{n=1}^{N} \int_{0}^{\infty} x N \lambda_{H} d x}{\eta_{L}} \\
\Rightarrow \frac{\lambda_{L}}{\eta_{H}} & =\frac{\lambda_{H}}{\eta_{L}}
\end{aligned}
$$

Part 2: Part 1 shows that $\frac{1}{N \lambda_{L}} F_{n}^{H}(x)-x$ is constant, then

$$
\begin{gathered}
\frac{1}{N \lambda_{L}} F_{n}^{H}(0)-0=\frac{1}{N \lambda_{L}} F_{n}^{H}\left(s_{i}\right)-s_{i} \\
\Rightarrow s_{i}=\frac{1}{N \lambda_{L}} .
\end{gathered}
$$

Part 3: Firm H's budget constrain can be expressed as ${ }_{1}$

Equation (B-2) shows that

$$
\sum_{n=1}^{N} \int_{0}^{\frac{1}{N \lambda_{L}}} x d F_{n}^{H}(x)=\eta_{H} B
$$

Substituting (B-4) to (B-3) yields

$$
d F_{n}^{H}(x)=N \lambda_{L} d x
$$

$$
\begin{aligned}
& \sum_{n=1}^{N} \int_{0}^{\frac{1}{N \lambda_{L}}} x N \lambda_{L} d x=\eta_{H} B . \\
& \text { Then } \lambda_{L}=\frac{1}{2 \eta_{H} B} .
\end{aligned}
$$

\section{Proof of Proposition 6}

Suppose the opposite. That is, a pure location strategy exists and is denoted by $\left(x_{H^{*}}, x_{L^{*}}\right)$.

Let Firm $\mathrm{H}$ stay on the left of Firm L and $x$ be the location of a consumer who feels indifferent in purchasing from Firm $\mathrm{H}$ or from Firm $\mathrm{L}$. Then the consumer's utility function satisfies: $\eta_{0}+\eta-\left(x-x_{H}\right)^{2}=\eta_{0}-$ $\left(x-x_{L}\right)^{2}$, from which $x=\frac{x_{L}{ }^{2}+\eta-x_{H}{ }^{2}}{2\left(x_{L}-x_{H}\right)}$ is derived.

The attractions of both firms are as follows: $T_{H}=x, T_{L}=1-x$. Moreover, $\eta<\left(x_{L}-x_{H}\right)\left(2-x_{L}-x_{H}\right)$ to ensure that the capability gap is not sufficient large to drive Firm $L$ out of marke.t 
Both firms maximize their attractions. Taking the first order of Firm H's profit function, $\frac{d T_{H}}{d x_{H}}=0$, yields:

$$
x_{L}^{2}+x_{H}^{2}=2 x_{H} x_{L}-\eta \text {. }
$$

Similarly, taking the first order of Firm B's profit function, $\frac{d T_{L}}{d x_{L}}=0$, yields:

$$
x_{L}^{2}+x_{H}^{2}=2 x_{H} x_{L}+\eta \text {. }
$$

Equations (B-6) and (B-7) are contradictory, so a pure strategy does not exist.

\section{Copyrights}

Copyright for this article is retained by the author(s), with first publication rights granted to the journal.

This is an open-access article distributed under the terms and conditions of the Creative Commons Attribution license (http://creativecommons.org/licenses/by/4.0/). 